\title{
Colloids as model systems for problems in statistical physics
}

\author{
D. Babič \\ 2. Physikalisches Institut, Universität Stuttgart, Pfaffenwaldring 57, 70550 Stuttgart, Germany \\ and Faculty of Mathematics and Physics, University of Ljubljana, Jadranska 19, 1000 Ljubljana, Slovenia \\ C. Schmitt and C. Bechinger \\ 2. Physikalisches Institut, Universität Stuttgart, Pfaffenwaldring 57, 70550 Stuttgart, Germany
}

(Received 13 September 2004; accepted 5 November 2004; published online 17 June 2005)

\begin{abstract}
Owing to their mesoscopic length scales, colloidal suspensions provide ideal model systems suitable for addressing many problems in the field of statistical physics. Exemplarily, we highlight the versatile nature of such systems by discussing experiments with stochastic resonance and a practical realization of a recently proposed ratchet cellular automaton. (C) 2005 American Institute of Physics.
\end{abstract}

[DOI: $10.1063 / 1.1839311]$

Colloidal systems, i.e., micron-sized particles which are suspended in liquids, share many properties with atomic systems. Therefore, they are often referred to as model systems to address novel concepts in the context of statistical physics in a convenient way. Owing to the Brownian motion of such particles, their trajectories can be experimentally studied and allow direct comparison with numerical and theoretical investigations. Here we report on two examples to highlight the use of colloidal particles. The first example is devoted to stochastic resonance, i.e., amplification of small periodic signals in a double-well potential in the presence of noise. The second example addresses a new type of a ratchet cellular automaton which has been recently suggested as a basic element in novel computing schemes.

\section{INTRODUCTION}

Molecules and atoms are always in thermal motion thereby continuously swarming and colliding with each other. While it is difficult to directly observe this motion in experiments, it can be visualized with micron-sized particles suspended in a liquid. Due to its bombardment by molecules of the liquid, a mesoscopic particle undergoes a Brownian motion which can be directly observed with a conventional microscope. When the Scottish botanist Robert Brown (1773-1858) performed his famous experiments, he used pollen from Clarkia Pulchella whose cytoplasm contained particles of about $5 \mu \mathrm{m}$ in size. ${ }^{1}$ Such particles, which are large compared to the molecules of the solvent so that the latter can be regarded as a homogeneous background but are small enough to exhibit Brownian motion, are known as colloids.

At the beginning of the 20th century, Jean Perrin performed a simple yet brilliant experiment demonstrating that the Brownian motion of a colloidal particle is just the largescale manifestation of the thermal agitation of the molecules in the liquid. This means that the energy equipartition theorem, i.e., the fact that the total mean translational kinetic energy of a molecule equals $3 / 2 k_{\mathrm{B}} T$ holds for the molecules of the liquid as well as for the colloids. In 1926, Perrin was awarded the Nobel Prize for this observation which is the cornerstone for the concept of using colloidal particles as model systems for problems in statistical physics.

With sizes comparable to the wavelength of visible light, colloidal systems can be conveniently investigated with optical methods. This allows to obtain direct positional information of individual particles. Apart from the length scale which distinguishes colloids from atoms by several orders of magnitude this also applies for the characteristic time scales. As a consequence, length and time scales completely separate in both systems. This is easily understood when we compare the time $\tau_{a}$ it takes an isolated particle to diffuse in a medium with viscosity $\eta$ over a distance comparable to its radius $a$

$$
\tau_{a}=\frac{a^{2}}{6 D_{\mathrm{SE}}}
$$

where $D_{\mathrm{SE}}$ describes the well-known Stokes-Einstein diffusion coefficient

$$
D_{\mathrm{SE}}=\frac{k_{B} T}{6 \pi \eta a} .
$$

For atoms $\tau_{a} \approx 1$ ps while for colloids the above expression yields $\tau_{a}$ on the order of seconds. This is also observed experimentally. Dynamical processes in colloidal systems are thus considerably slower compared to atomic systems as illustrated by, e.g., the dynamics of glass formation. While in metallic systems this typically takes place in times on the order of $10^{-13}$ seconds it occurs on time scales of several seconds or even minutes in colloidal systems. This allows to study the dynamical aspects of this interesting transition.

Owing to their Brownian motion, colloidal particles sample their accessible configurational space. In that sense colloids can be regarded as "computer simulator's dreams" because they allow real time "calculations" in complex situations. ${ }^{2}$ Since the pair-interaction in colloidal systems can be tuned over a large range, colloids are considered as versatile model systems. During recent years growing interest in colloidal systems in the presence of external fields has arisen. ${ }^{3}$ In this context optical trapping techniques (optical 
tweezers) are particularly useful because they allow to manipulate micrometer- and sub-micrometer sized particles in a non-destructive way. ${ }^{4-7}$ This makes optical tweezers a flexible tool which has been successfully applied in many experiments.

The principle of optical trapping is based on the transfer of momentum from photons (typically emitted from a laser source) which are reflected and refracted by a trapped particle to the particle itself. ${ }^{4,8}$ The net effect is that colloids with a higher index of refraction than the surrounding liquid are pushed toward regions of highest light intensity, i.e., toward the focus of the light beam. Optical forces acting on colloidal particles are usually divided into two types: the scattering force and the gradient force. The scattering force is proportional to the light intensity and acts in the direction of the light propagation (light pressure) whereas the gradient force is proportional to the spatial gradient of the light intensity and acts in the direction of the intensity gradient.

Instead of using a single focused laser beam one can also apply spatially extended light patterns to a colloidal suspension. Such stationary patterns can be either created by interfering laser beams ${ }^{9,10}$ or by holographical arrays. ${ }^{11}$ In case of two-dimensional colloidal systems light patterns can be used to mimic periodic substrate potentials. ${ }^{9,10,12}$

When the focus of the beam moves on time scales very short compared to typical relaxation or diffusion times in colloidal systems, quasi-static light-potentials are created. This allows to study the motion of colloids in complex external fields. Experimentally, such scanned optical tweezers can be realized, e.g., by galvanostatic mirror units, where an incoming laser beam is reflected by adjustable small mirrors. Due to the inertia of the mirrors, however, repetition rates above $300 \mathrm{~Hz}$ are difficult to achieve. Much higher speeds can be obtained using acousto-optical deflectors (AOD), where an incoming laser beam is deflected on a density wave excited inside a transparent crystalline material. Since the wavelength of the density wave can be changed within several microseconds, scanning rates up to $100 \mathrm{kHz}$ are possible. In this paper we report on two examples to highlight the use of colloidal particles for investigations in the field of statistical physics. The first example describes an experiment on stochastic resonance (SR) of a colloidal particle in an optically created double-well potential. SR is believed to be responsible for an amazingly wide range of phenomena such as the periodic occurrence of ice ages, the feeding behavior of paddlefish and even human balance control and visual perception (for a review see, e.g., Ref. 13). The essential characteristic of SR is that it improves the detection of weak periodic signals in nonlinear systems by the presence of noise. The second example is a practical realization of a recently proposed ratchet cellular automaton (RCA). ${ }^{14}$ Such a device has been suggested as a possible element in future computing schemes but RCA also have several interesting statistical properties, when operated in a nondeterministic regime.

\section{EXPERIMENTAL SETUP}

The experimental setup was composed of three main parts-an optical tweezer, ${ }^{8}$ a sample cell with deionizing cir- cuit and a data acquisition system. The optical tweezer was constructed around a custom built microscope with two coaxially placed, vertically oriented microscope objectives in a confocal configuration and a sample cell containing colloidal particles. The laser beam was focused by the upper objective $(50 \times, N A=0.6)$ which also served as a condenser for the particle illumination. The lower objective $(40 \times, N A=0.5)$ together with a low pass optical filter blocking the laser light was used to image the particles onto a CCD camera. As laser source we used the $\mathrm{TEM}_{00}$ output of a frequency doubled solid state laser at $532 \mathrm{~nm}$. The beam was first expanded to a diameter of $\sim 3.5 \mathrm{~mm}$, guided through a two axis acoustooptic deflector (AOD) and finally inserted into the entrance aperture of the upper objective by a telecentric lens system. Together with a custom built driving electronics and control software, the AOD enabled us to create multiple optical traps by time sharing the laser beam with a switching rate of $50 \mathrm{kHz} .{ }^{15}$ The range of the optical tweezers in the sample plane was $150 \times 150 \mu \mathrm{m}$, with nanometer resolution and repeatability on the order of $100 \mathrm{~nm}$. The intensity of each trap could be set with 12-bit resolution. Measurements were done with 1.2 and $2.4 \mu \mathrm{m}$ diameter polystyrene particles obtained from IDC. A highly diluted suspension was inserted into a sample cell via a standard deionizing circuit described elsewhere. ${ }^{16}$ All experiments were performed at room temperature with a stability of $\pm 1 \mathrm{~K}$. Complex light potentials were created by placing optical traps at different positions. Due to time sharing of the laser beam between the traps possible interference effects were absent. The switching time between the traps was $20 \mu \mathrm{s}$, so the potentials can be regarded as quasistatic. While the gradient light force induced only the lateral confinement of the particles, they were confined in the vertical direction by the light pressure of the perpendicular incident laser beam. This component caused the colloids to be pushed towards the lower wall of the sample cell where it was counterbalanced by the electrostatic repulsion leading to an equilibrium distance of $\sim 500 \mathrm{~nm}$. Effectively, our system was thus confined to two dimensions. The particle motions were recorded with a CCD camera connected to a PC installed frame grabber. Data were acquired at 20 frames per second and recorded in a compressed format onto a hard drive. Proprietary software was used for particle recognition and trajectory reconstruction. For a review of this technique we refer to the literature. ${ }^{17}$

\section{STOCHASTIC RESONANCE}

Most formal descriptions of SR typically consider the one-dimensional motion of an overdamped particle in a double-well potential coupled to a thermal reservoir at temperature $T .{ }^{18}$ The particle performs thermally activated jumps across the barrier characterized by the Kramers time ${ }^{19} T_{K}$ $=T_{R} \exp (\Delta V / D)$. Here $\Delta V$ is the potential barrier and $D$ the noise strength (i.e., the thermal energy in our case) and $T_{R}$ the intrawell relaxation time. If the double-well potential is periodically tilted by applying a weak external force with period $T_{\Omega}$, the transition probabilities of the thermally activated hops become time dependent. In this context a weak force means that in the absence of thermal fluctuations ( $T$ 


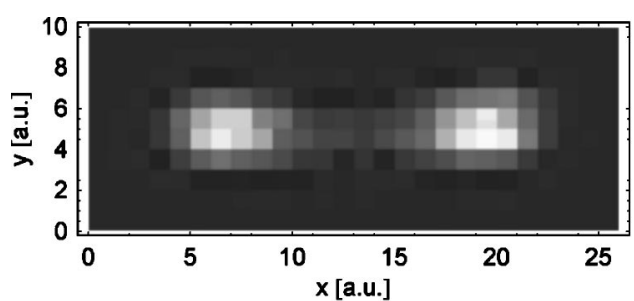

(a)

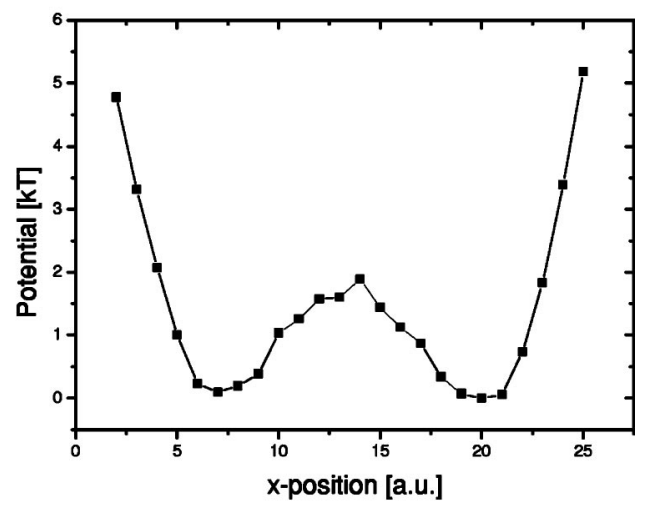

(b)

FIG. 1. (a) Grayscale representation of the two-dimensional probability distribution of a colloidal particle fluctuating in a static double-well potential created by optical tweezers. (b) Cross section of the double-well potential for $y=5$ obtained from the probability distribution shown in (a). One clearly sees that the potential is rather symmetric.

$=0 \mathrm{~K})$ no barrier crossings take place. It can be shown that if the two time scales match, i.e., if $T_{\Omega} \approx 2 T_{K}$, a maximum synchronization between the thermally activated hopping and the weak periodic driving of the system occurs. Since the Kramers time is directly related to the noise strength $D$, the above condition is met at some optimum noise level. As a result the synchronization which can be quantified by, e.g., the signal-to-noise ratio displays a maximum as a function of $D$ being one of the most striking characteristics of SR. It is important to realize that instead of tuning $D$, the matching condition can be also fulfilled by adjusting $T_{\Omega}$. This is particularly useful in systems where the noise is intrinsic (as is the case in our experiments). In this case it is more convenient to characterize SR via an analysis based on the residence time distribution $P_{\text {res }}(t)$, quantifying the probability that a particle stays over the time $t$ within a certain potential well.

Figure 1(a) shows the two-dimensional particle probability distribution in an unmodulated light potential created by two laser traps. ${ }^{20}$ From these data we obtained the shape of the potential as shown in Fig. 1(b). As can be seen the cross section of the potential corresponds to a symmetric doublewell with a barrier height of about $2 k_{B} T$. To obtain the residence time distribution from the $x$-component of the particle trajectory (Fig. 2) we determined the time intervals between passages of the particle over suitably defined thresholds. In the absence of modulation, $P_{\text {res }}(t)$ decays exponentially for times larger than the intrawell relaxation time $T_{R}$ (being on the order of $0.1 \mathrm{~s}$ as estimated from the decay of the auto-

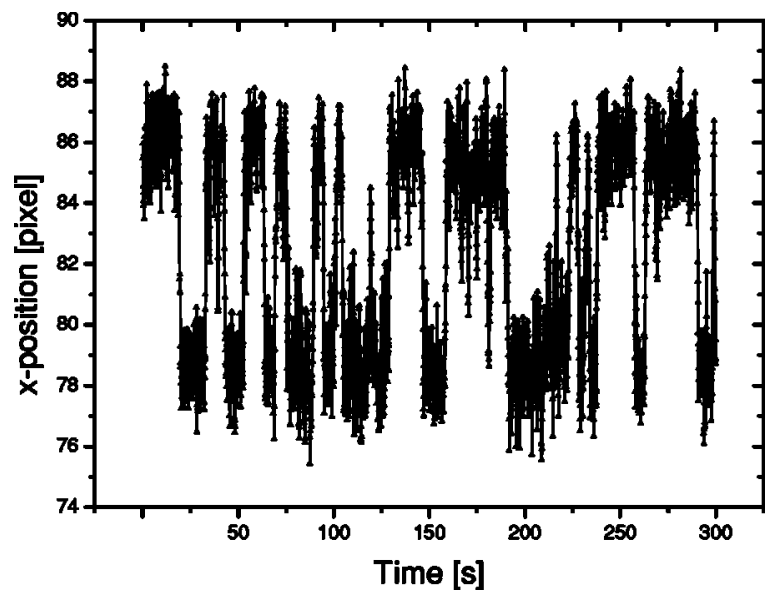

FIG. 2. Section of the $x$-component of the trajectory of a colloidal particle which is confined to a symmetric double-well potential. The data show the random transitions of the particle between the two potential wells shown in Fig. 1(b).

correlation function of the intrawell particle motion) with the decay constant given by $T_{K}$. For our experimental parameters $T_{K}$ was measured to be $7.3( \pm 0.4) \mathrm{s}$. In the presence of a periodic modulation, $P_{\text {res }}$ shows significant deviations from an exponential decay as is exemplarily demonstrated in Figs. 3(a)-3(c) for modulation periods $T_{\Omega}=2,14,40 \mathrm{~s}$. For better comparison we normalized $P_{\text {res }}$ with respect to the total number of events $\left[\Sigma P_{\text {res }}(t)=1\right]$ and plotted it vs the dimensionless time $t / T_{\Omega}$. Modulation times smaller than $T_{K}$ lead to the distribution with a series of peaks centered at odd multiples of $T_{\Omega} / 2$ [Fig. 3(a)]. These peaks appear due to the timedependent transition probabilities between the potential wells. The separation of the peaks becomes obvious when considering that on average a particle "waits" until the opportunity for a hop is largest. If the particle misses this opportunity it will reside in the same well for another (or mul-

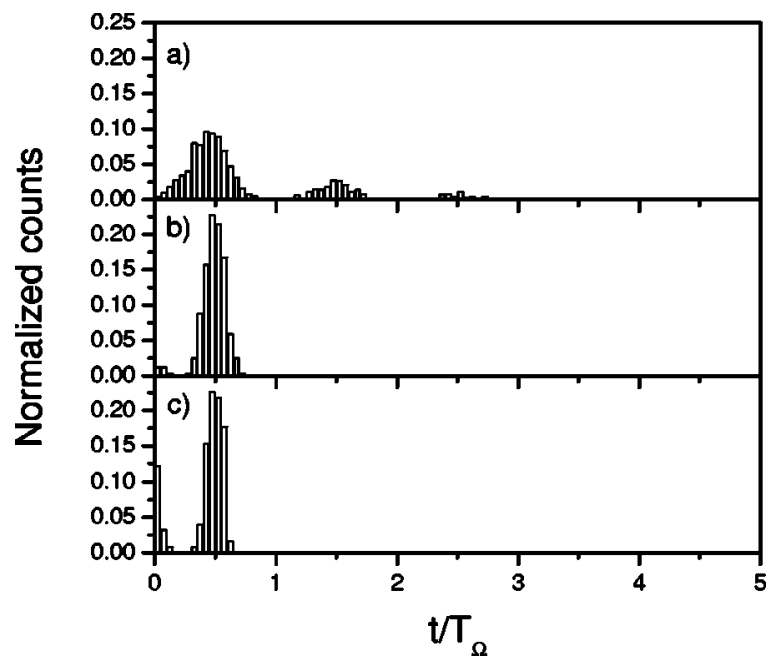

FIG. 3. Normalized residence time distributions as a function of time $t / T_{\Omega}$ for (a) $T_{\Omega}=2$, (b) 14 , and (c) $40 \mathrm{~s}$ for asymmetric modulation with an intensity modulation amplitude of $\pm 15 \%$. One clearly observes that close to the resonance condition, i.e., $T_{\Omega} \approx 2 T_{K}$ which holds for curve (b), most of the hopping events are synchronized with the external potential modulation. This behavior is characteristic for SR. 


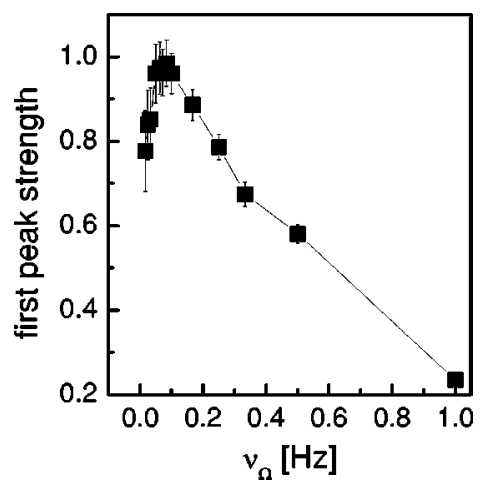

FIG. 4. Strength of the first peak area vs modulation frequency $\nu_{\Omega}=1 / T_{\Omega}$ for a modulation amplitude of $\pm 15 \%$. The curve shows a clear maximum thus demonstrating SR in our system.

tiple) $T_{\Omega}$. The position of the first peak follows from the fact that there are at most two "good" opportunities per modulation period for the particle to hop. The observed structure of $P_{\text {res }}$ is in very good agreement with numerical calculations. ${ }^{21}$ Because thermally activated barrier crossings are statistically independent, the peak height is predicted to decay exponentially $^{21}$ which is also in agreement with our experimental findings.

Upon increasing $T_{\Omega}$ towards the SR condition, the multipeak structure disappears and the residence time distribution becomes narrower until essentially only a single peak at $T_{\Omega} / 2$ is observed [Fig. 3(b)]. In this situation, the stochastic barrier crossing of the particle is maximally synchronized with the periodic modulation. ${ }^{22}$ When $T_{\Omega}$ is much higher than $T_{K}$, the particle hopping becomes less synchronized again which can be seen by the increasing transition probability at short times [Fig. 3(c)].

In order to demonstrate $\mathrm{SR}$ in systems where the noise strength remains constant, Gammaitoni et al. suggested to plot the strength (i.e., area) of the $n$th peak in $P_{\text {res }}$ as a function of $\nu_{\Omega}=1 / T_{\Omega} \cdot{ }^{23-25}$ In case of SR, the curve should exhibit a pronounced maximum. We applied this criterion to our data although the system is not truly one-dimensional as assumed in Ref. 23. Figure 4 shows the first peak strength as a function of $\nu_{\Omega}$ which indeed displays a maximum thus definitely demonstrating SR in a colloidal system.

\section{RATCHET CELLULAR AUTOMATA}

Despite tremendous achievements in the design and fabrication of microelectronic devices their operation still relies on the originally proposed physical mechanisms. There is, however, a growing interest in different approaches to further increase the speed and circuit density. In quantum cellular automata (QCA) information is encoded as positional or orientational configurations of interacting (quasi) particles or spins. $^{26-29}$ In most QCA schemes logic operations are performed adiabatically with the system remaining close to its ground state. This severely limits their speed and requires large energy separations (or low operation temperatures) between the ground and excited states for reliable operation. To avoid these drawbacks, recently a novel ratchet-like mechanism in a ratchet cellular automaton (RCA) has been sug- gested. Such elements allow to perform data transfer and logic operations in a classical system of coupled particles in a clocked fashion which is superior to current concepts of cellular automata operated in thermal equilibrium. ${ }^{14}$ Here we present the first experimental realization of a RCA in a colloidal system.

The experimental construction of a ratchet cellular automaton requires the arrangement of many interacting colloidal particles in a linear chain-like fashion as shown in Fig. 5(a). Each particle is located in a double-well light potential oriented perpendicularly to the chain orientation. In this geometry the particle position is essentially restricted to two states (up-down-see Fig. 5). Due to the repulsive particle interaction the ground state of the system corresponds to the zigzag configuration. Such a structure can be used as an information processing device, the most basic being a simple transmission line. A bit of information is represented by a defect where two neighboring particles are in the same state (i.e., up-up or down-down). Such a defect should be propagated along the chain in one direction, preferably in a clocked way. This can be achieved by a ratcheting mechanism, ${ }^{14}$ where within local groups of three particles [denoted by $A, B$, and $C$ in Fig. 5(a)], the inter-particle coupling is periodically modulated. The coupling is varied by moving the potential double-wells together with the associated particles in the direction along the chain. The clock period consists of three phases I, II, and III which are schematically depicted in Fig. 5(b). For the sake of clarity we additionally introduced intermediate steps within the single phases (e.g., I/ $a$ and I/ $b$ ). Starting with phase I/ $a$, the intersite distances $B-C$ and $C-A$ are equal and slightly smaller than $A-B$. A defect introduced at the left end of the chain (phase $\mathrm{I} / b$ ) is stable since particle $B_{1}$ (comprising together with particle $A_{1}$ a defect) is more strongly coupled to its right neighbor $C_{1}$ than to $A_{1}$. At the clock transition to phase II all the $B$ sites are moved closer to the $A$ sites [phase II/ $a$ on Fig. $5(\mathrm{~b})]$, simultaneously increasing the $B-C$ distances. In this situation, it is energetically favorable for particle $B_{1}$ to switch its position transfering the defect by one step to the right (phase II $/ b$ ). The transition to phase III is achieved by moving sites $C$ closer to sites $B$ and by the same effect advancing the defect one step further to the right (phases III/ $a$ and III $/ b$ ). The starting configuration is restored at the next clock transition by simultaneously moving sites $B$ and $C$ back to their original positions of phase I. In doing so the defect is propaged another step and is now located within the next $A B C$ group. Applying the prescribed clock sequence periodically will propagate a defect along the chain.

Experimentally the above described RCA was realized by fast AOD-based laser tweezers used to create up to forty double-well potentials arranged in the linear chain. All double-wells were occupied by $2.4 \mu \mathrm{m}$ diameter latex particles with high surface charge density. The average distance between the double-wells was $3.5 \mu \mathrm{m}$ and the well separation was $2.5 \mu \mathrm{m}$. Each double-well was comprised of three laser traps, the middle one providing a direct control of the barrier height. Inter-particle coupling was controlled either by varying the average distance between the chain locations or by adjusting the salt concentration and henceforth the 


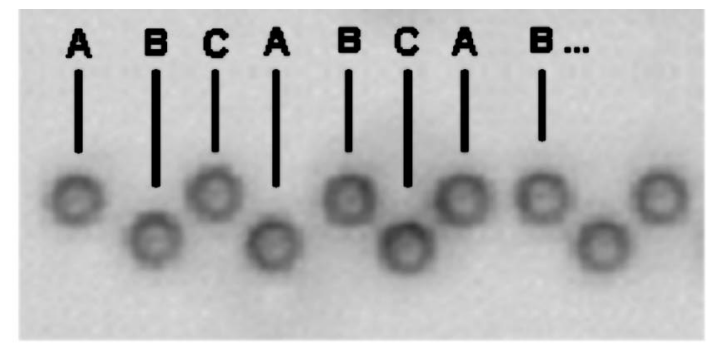

(a)

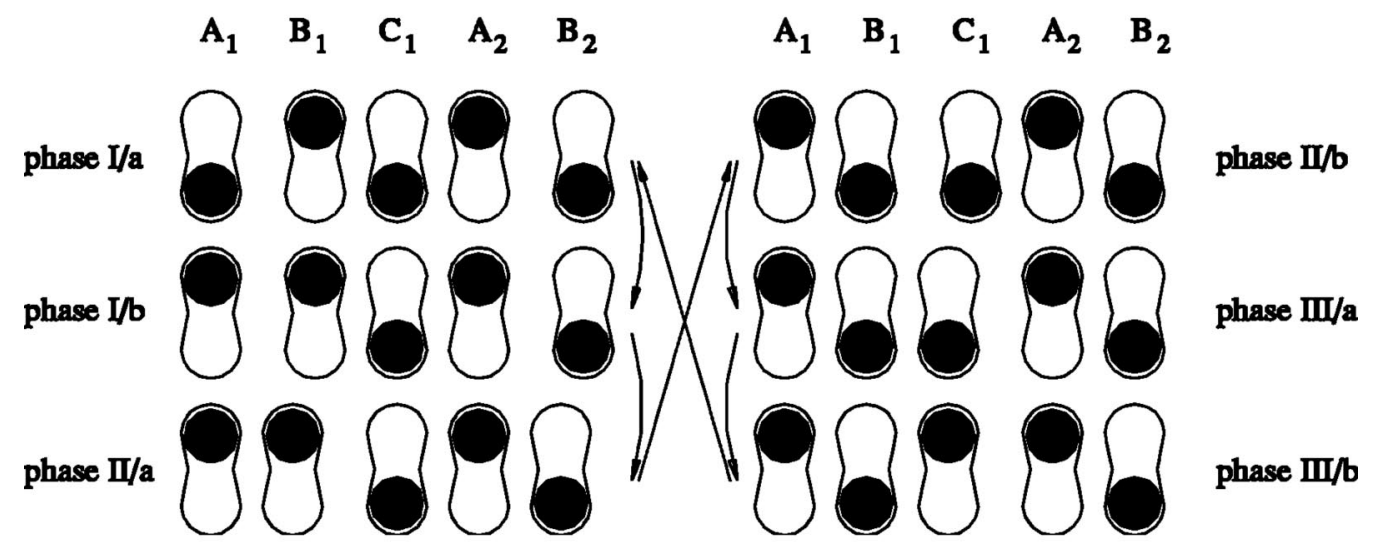

(b)

FIG. 5. (a) Part of the ratchet chain composed of colloidal particles (laser traps are not visible). Letters A, B, and C denote groups within which a three phase clock modulation is performed to allow the directed transport of defects within the chain. (b) Schematic depiction of a three phase clock modulation which can propagate a defect through a linear chain of interacting particles. The thin closed curves represent the confining sites which were occupied with colloidal particles (dark circles). Each clock phase is divided into two parts ( $a$ and b), which correspond to the configuration before and after the particles changed their positions within the confining sites.

screening length of the electrostatic repulsion. Flexibility of the AOD deflection system together with proprietary control software provided a means for creating the above described three phase clocked modulation necessary for the ratchet operation. For a properly operating ratchet the typical clock period was a few seconds and the amplitude of the modulation was $0.7 \mu \mathrm{m}$. The defects were introduced at the left end of the chain, by switching the leftmost particle's position with a separate laser trap.

The working of the ratchet was analyzed with methods similar to the ones used in the SR experiment. From the recorded trajectories hops of individual particles were identified so the defects could be tracked as they traveled along the chain. Figure 6 shows the case where defects were periodically introduced into the ratchet. It follows from the principles of the ratchet operation that a defect can only be accepted by the ratchet at the clock phase II when the inter-site distances $A-B$ are smaller than $B-C$. As a consequence the defects that enter the chain are unevenly spaced even though the input particle is switched evenly. This is confirmed by the experiment and is clearly indicated by two peaks in the inset of Fig. 6, showing a waiting time distribution of the last particle (output). The same plot for the input particle only has one peak (not shown). Phase dependent defect acceptance at the input also leads to the upper frequency limit for the defect introduction, imposed by the clock speed. Apart from operating the ratchet as a simple transmission line we also experimentally verified the possibility of combining more coupled chains to perform logical operations as proposed in Ref. 14 (data not shown).

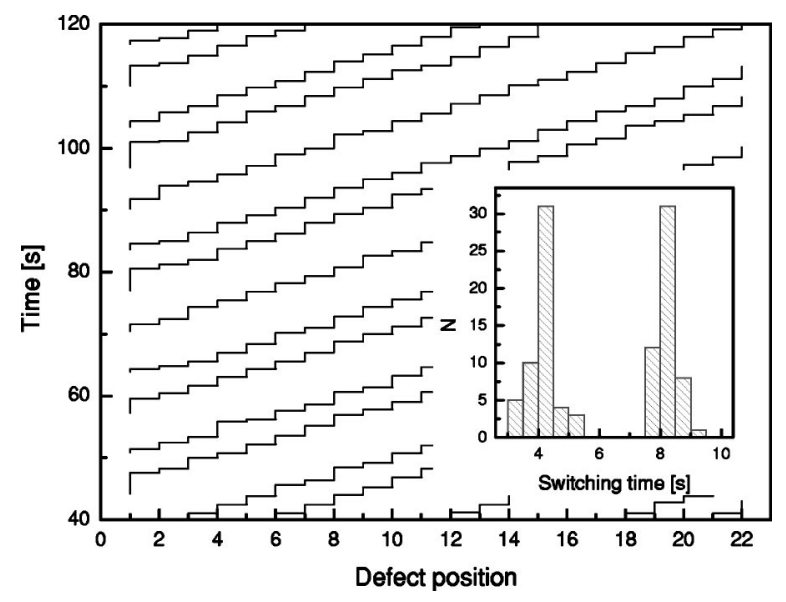

FIG. 6. Defect propagation along the ratchet chain. Clock period was $4 \mathrm{~s}$ and defects were introduced periodically with the period of $6.5 \mathrm{~s}$. In the inset a waiting time distribution of the last chain particle (output) is shown. Two peaks indicate a clock phase dependant acceptance of the defect at the input, and the peak width is a consequence of the Brownian noise which makes particles' switching uneven. 


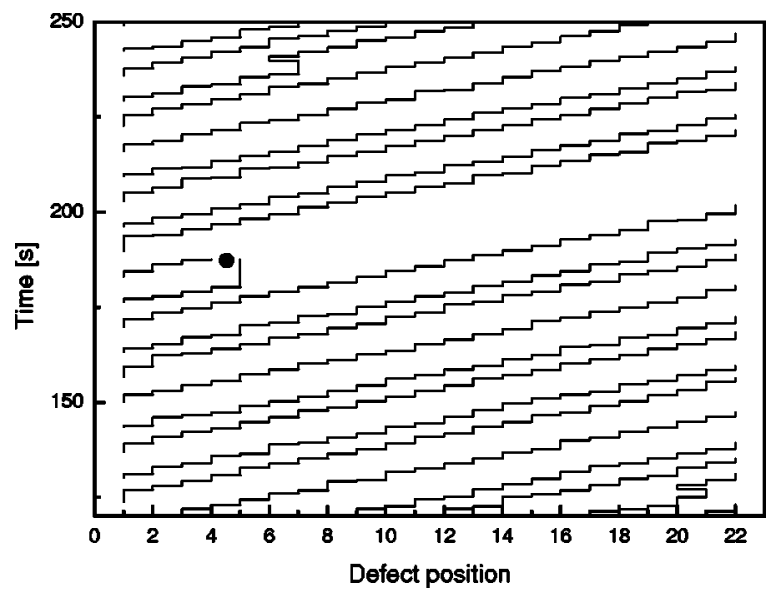

FIG. 7. Defect propagation in the ratchet operated in a nondeterministic regime. Occasional stalling or back stepping is evident. At the position denoted by dot, two trajectories disappear which corresponds to the annihilation of a pair of defects.

For computation purposes a clocked operation of the ratchet is desired. However, a finite width of the peaks in Fig. 6 indicates that position switching of the particles along the chain, as the defect gets propagated, is uneven. This is due to the particles' thermal motion and was also predicted by the simulation. ${ }^{14}$ By varying, for example, the height of the potential barrier of the double-wells this effect can either be reduced or increased. The latter ultimately drives the operation of the ratchet into a nondeterministic regime. This happens whenever the particles' hopping over the barrier of the double-wells is essentially assisted by the thermal motion. A finite probability for the particle not to hop even when the clock phase dictates so, leads to the stalling of the defect propagation and sometimes even back stepping. Such occurrences are shown in Fig. 7. Whenever two defects catch on each other they annihilate (also shown in Fig. 7). There are many parameters which affect the passage of the ratchet from deterministic to nondeterministic regime, i.e., barrier height, coupling strength, overall confinement of the particles in their potential wells, clock speed, etc. So far only the existence of the nondeterministic mode has been experimentally verified, but the number of controllable parameters and pathways of defect propagation and annihilation indicate a potentially very rich behavior of the ratchet which eventually may also be useful for the design of improved concepts for cellular automata.

In summary, we have demonstrated the use of colloidal systems for addressing two issues in the field of statistical physics. First, we demonstrated the occurrence of stochastic resonance of a colloidal particle in a periodically driven bistable potential. We observed that if the driving frequency matches the time scale characteristic for statistical barrier crossing of the particle, a maximum synchronization between the particle hopping and the external potential modulation occurs. In the second example we presented the experimental realization of a clocked ratchet cellular automaton and demonstrated the principle of operation as recently suggested by numerical simulations. Both examples underline the successful application of colloidal suspensions for the demonstration of the validity of novel concepts developed for classical systems.

\section{ACKNOWLEDGMENTS}

We acknowledge helpful discussions with Peter Reimann and Peter Hänggi. This work is financially supported by the Deutsche Forschungsgemeinschaft (BE 1788/ 4-1).

${ }^{1}$ R. Brown, Edinburg J. Science 1, 314 (1829).

${ }^{2}$ D. Frenkel, Physica A 313, 1 (2002).

${ }^{3}$ H. Löwen, J. Phys.: Condens. Matter 13, R415 (2001).

${ }^{4}$ A. Ashkin, J. M. Dziedzic, J. E. Bjorkholm, and S. Chu, Opt. Lett. 11, 288 (1986).

${ }^{5}$ A. Resnick, J. Colloid Interface Sci. 262, 55 (2003).

${ }^{6}$ D. G. Grier, Nature (London) 424, 810 (2003).

${ }^{7}$ K. C. Neuman and S. M. Block, Rev. Sci. Instrum. 75, 2787 (2004).

${ }^{8}$ A. Ashkin, Phys. Rev. Lett. 24, 156 (1970).

${ }^{9}$ M. Brunner and C. Bechinger, Phys. Rev. Lett. 88, 248302 (2002).

${ }^{10}$ J. Baumgartl, M. Brunner, and C. Bechinger, Phys. Rev. Lett. 93, 168301 (2004).

${ }^{11}$ K. Mangold, P. Leiderer, and C. Bechinger, Phys. Rev. Lett. 90, 158302 (2003).

${ }^{12}$ M. Brunner and C. Bechinger, Prog. Colloid Polym. Sci. 123, 156 (2003).

${ }^{13}$ P. Hänggi, ChemPhysChem 3, 285 (2002).

${ }^{14}$ M. B. Hastings, C. J. O. Reichhardt, and C. Reichhardt, Phys. Rev. Lett. 90, 247004 (2003).

${ }^{15}$ K. Visscher, S. P. Gross, and S. M. Block, IEEE J. Quantum Electron. 2, 1066 (1996).

${ }^{16}$ D. Rudhardt, C. Bechinger, and P. Leiderer, Phys. Rev. Lett. 81, 1330 (1998).

${ }^{17}$ J. C. Crocker and D. G. Grier, J. Colloid Interface Sci. 179, 298 (1996).

${ }^{18}$ L. Gammaitoni, P. Hänggi, P. Jung, and F. Marchesoni, Rev. Mod. Phys. 70, 223 (1998).

${ }^{19}$ P. Hänggi, P. Talkner, and M. Borkovec, Rev. Mod. Phys. 62, 251 (1990).

${ }^{20}$ D. Babic, C. Schmitt, I. Poberaj, and C. Bechinger, Europhys. Lett. 67, 158 (2004).

${ }^{21}$ T. Zhou, F. Moss, and P. Jung, Phys. Rev. A 42, 3161 (1990).

${ }^{22}$ P. Jung and P. Hänggi, Phys. Rev. A 44, 8032 (1991).

${ }^{23}$ L. Gammaitoni, F. Marchesoni, and S. Santucci, Phys. Rev. Lett. 74, 1052 (1995).

${ }^{24}$ M. H. Choi, R. F. Fox, and P. Jung, Phys. Rev. E 57, 6335 (1998).

${ }^{25}$ F. Marchesoni, L. Gammaitoni, F. Apostolico, and S. Santucci, Phys. Rev. E 62, 146 (2000).

${ }^{26}$ I. Amlani, A. O. Orlov, G. Toth, G. H. Bernstein, C. S. Lent, and G. L. Snider, Science 284, 289 (1999).

${ }^{27}$ R. P. Cowburn and M. E. Welland, Science 287, 1466 (2000).

${ }^{28}$ A. O. Orlov, I. Amlani, G. H. Bernstein, C. S. Lent, and G. L. Snider, Science 277, 928 (1997).

${ }^{29}$ C. S. Lent and B. Isaksen, IEEE Trans. Electron Devices 50, 1890 (2003). 